\title{
SULM - Die Chance für eine rationale (Labor-)Medizin
}

\author{
A. R. Huber
}

\section{Zusammenfassung}

Die Schweizerische Union für Labormedizin (SULM) hat als Dachverband aller laborinteressierten Kreise wichtige Aufgaben. Darunter Unterstützung bei der Einführung von Qualitätssicherungsprogrammen wie Akkreditierung, Förderung der wissenschaftlichen Dokumentation über den medizinischen und ökonomischen Nutzen von Labormedizin, hierbei insbesondere, inwiefern durch gezielte Diagnostik die Kosten im Gesundheitswesen kontrolliert werden können. Weiter bemüht sich die SULM um Koordination und Nutzung von Synergien der verschiedenen Interessenten aus diesem Bereiche des Gesundheitswesens, wobei auch eine transparente, unabhängige Kooperation mit der Industrie unterstützt wird. Im folgenden werden diese Aspekte erläutert, wie auch die Entwicklungstendenzen in der Medizin bezüglich Effektivität und Effizienz, sowie hinsichtlich Qualität und auch ethische Aspekte ausgeführt.

Analog zu den vorbildlichen chirurgischen Disziplinen wurde vor elf Jahren die Schweizerische Union der Labormedizin (SULM) gegründet. Als deren vierter Präsident seien mir einige Bemerkungen erlaubt.

\section{Zweck und Aufgaben}

Da die SULM von mehreren Standesorganisationen, legislativen Behörden und «tiers garant» sowie der Diagnostikindustrie paritätisch getragen wird, ergeben sich diesem Verbund aller an Laborfragen Interessierten verständlicherweise übergeordnete, gemeinsame Aufgaben. Prinzipiell geht es hier darum, die Labormedizin als junges Mitglied der bunten medizinischen Palette bekannt zu machen und zu fördern. Spezifisch sind aber folgende statutarische Aufgaben festgehalten, nämlich:

Korrespondenz:

Andreas R. Huber

Präsident SULM

c/o Zentrum für Labormedizin

Kantonsspital Aarau

CH-5001 Aarau

Tel. 0628385302

Fax 0628385399

E-Mail: andreas.huber@ksa.ch

\section{Zweck}

Die SULM vertritt als Dachverband die schweizerischen Institutionen und Verbände der Laboratoriumsmedizin, insbesondere gegenüber der Ärzteschaft, der Bevölkerung und den Behörden.
Als Verband setzt sich die SULM für eine effiziente, effektive und patientenbezogene Labormedizin ein. Zweifelsohne haben gezielt eingesetzte Laboranalysen einen kostensparenden Effekt. Dies auf wissenschaftlicher Basis und unter dem Aspekt, höchsten Qualitätsansprüchen der Patienten zu genügen. Da dies zu wenig bekannt ist, wird eine Arbeitsgruppe der SULM dies aufzeigen.

\section{Aufgaben}

- Unterstützung von Qualitätssicherungsprogrammen und Mithilfe bei der Akkreditierung und Zertifizierung nach EN/ISO-Normen.

- Förderung der wissenschaftlichen Dokumentation zum Nutzen (medizinisch, wissenschaftlich und für das Gesundheitssystem wirtschaftlich) der Laboratoriumsmedizin.

- Koordination der Tätigkeiten von Berufsverbänden, Industrie und Organisationen im Rahmen der Laboratoriumsmedizin und Zusammenarbeit mit Behörden und Organisationen.

\section{Qualitätssicherung}

In Anbetracht der Vorreiterrolle, welche die Labormedizin resp. die Laboratorien im Bereich der Qualitätssicherung hatten und immer noch haben, ist es eine wichtige Aufgabe der SULM, diese Aspekte weiter zu fördern - unter dem Gesichtspunkt einer international vergleichbaren, vernünftigen und praktikablen Umsetzbarkeit. Es ist wahrscheinlich nicht allen bekannt, dass die Schweiz mit ihren Laboratorien bei der Akkreditierung international eine Vorreiterrolle einnimmt. So hat die Schweiz absolut und auch relativ die grösste Anzahl akkreditierter Laboratorien. Korrekterweise wird diese Akkreditierung von einem unparteiischen, finanziell eigenständigen, unabhängigen eidgenössischen Amt (metas, metrologie und akkreditierung schweiz) umgesetzt, das international selbst akkreditiert ist. So sind in der Schweiz um die 80 Laboratorien akkreditiert oder im offiziellen Verfahren. 
Abbildung 1

Qualitätspyramide.

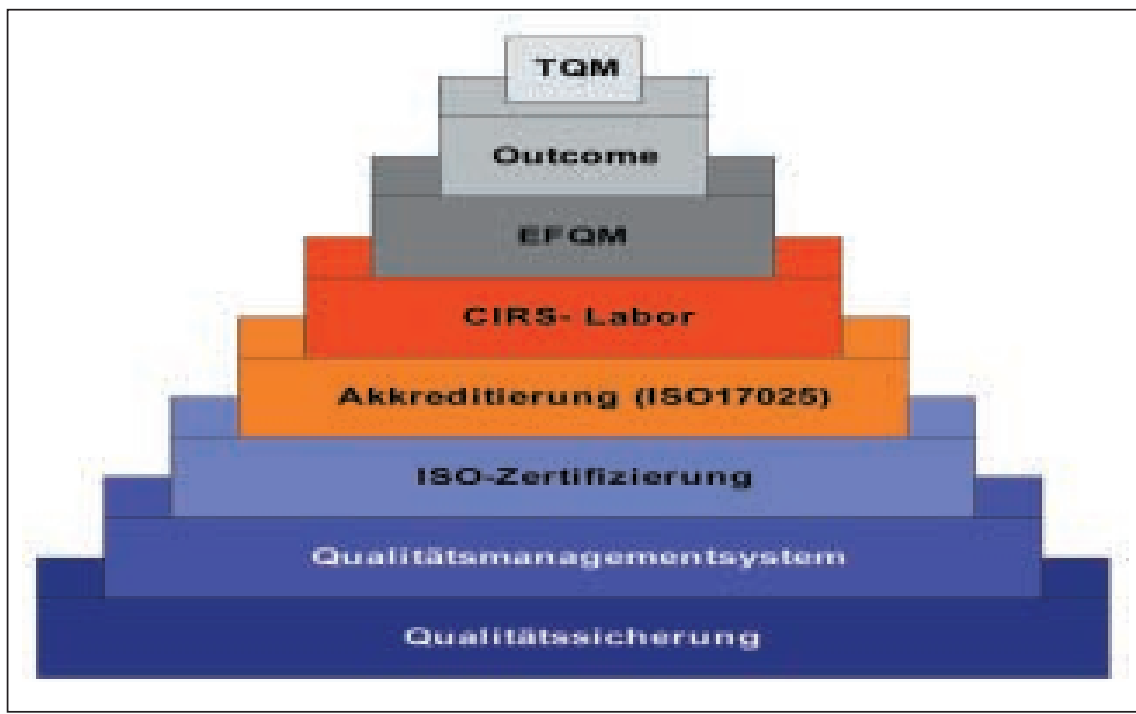

Aus den vielen möglichen Qualitätssicherungssystemen und Modellen wurden als breitbasig dastehende, solide, sämtliche Mitarbeiterschichten umfassende Systeme der ISO/EN-Norm (ISO 49001, 17025, 15189) gewählt. Bei diesen Systemen geht es nicht um prestigeträchtige Wettbewerbe und glitzernde Zertifikate, sondern um eine Qualität von der Basis, für die tägliche Arbeit und unabhängig von Galionsfiguren. Allerdings kann dabei ein ISO 9000 u.a. nicht genügen, da dies lediglich das Vorhandensein eines Qualitätssystems, aber keineswegs das Vorhandensein von Fachkompetenz und anderen viel wesentlicheren Aspekten belegt. Auch sind für die Labormedizin Modelle der EFQM, VQG, Outcome-Datensammlungen etc. zu selektiv, als dass sie für die Praxis als geeignete qualitätssichernde Massnahmen und Systeme bezeichnet werden könnten.

In den Augen der SULM sieht eine vernünftige Qualitätspyramide so aus, wie sie in der Abbildung 1 dargestellt ist.

Zusammenfassend, bedingt durch den «angeborenen» Drang zur Messbarkeit, misst die Labormedizin einer soliden Qualitätssicherung höchste Bedeutung zu. Demzufolge bemüht sich die SULM darum, dass die Akkreditierung oder mindestens ein analoger Standard zur Norm in der schweizerischen und hoffentlich auch in der internationalen Laborgemeinschaft wird.

\section{Internationale Kontakte}

Kaum zu glauben, aber im Ausland wird die Schweiz und im speziellen, und in diesem $\mathrm{Zu}$ sammenhang interessierend die SULM benieden. In Deutschland bestanden noch kürzlich Spannungen zwischen Labormedizinern und klinischen Chemikern, während die Grundausbildung der letzteren mehrheitlich in naturwissenschaftlichen Fächern erfolgte, dominiert bei den Labormedizinern das Medizinstudium. In Frankreich sind die Laboratorien von der Ärzteschaft weitgehend abgekoppelt, während in den USA mehrheitlich nur noch gigantische Riesenlabors vorhanden sind, die mit den Versicherern Rabattvereinbarungen haben und dem Arzt wie auch indirekt seinen Patienten wenig Spielraum erlauben. In unserem Land werden «nichtmedizinische» Naturwissenschafter ebenso hoch geachtet wie Mediziner. Obwohl es Unterschiede gibt, sind beide Parteien grosso modo gleichberechtigt. Während den Nichtmedizinern anfangs eher die klinische Kompetenz fehlt, mangelt es den Medizinern gelegentlich an präzisem, analytischem, naturwissenschaftlichem Denken. Für Mediziner ist sicher eine Vertiefung der naturwissenschaftlichen Ausbildung während des Studiums vonnöten, während auf der anderen Seite reine Naturwissenschafter wie Pharmazeuten, Biologen, Biochemiker u.a. eine zusätzliche Weiterbildung in gewissen medizinischen Belangen benötigen. Unter Berücksichtigung des oben Genannten setzt sich die SULM für die Aufnahme des FAMH-Titels in das neue Medizinalberufegesetz (MedBG) ein. Zweifelsohne sind die Schweizerischen Laborleiter (FAMH-Titelträger) im internationalen Vergleich hervorragend aus- und weitergebildet. Ein Zutritt in den europäischen Raum muss unbedingt ermöglicht werden. Weiter hat die SULM intensive Kontakte mit China, EuroMedLab mit MedLab 2003, sowie mit vielen internationalen Fachgesellschaften wie Alps-Adria, IFCC, DGLM, DGKC. Wir sind überzeugt, dass diese internationalen Kontakte für die Schweiz wie auch für die internationalen Partner fruchtbar sind.

\section{Effektivität und Effizienz}

Ein weiteres zentrales Anliegen der SULM ist die Effektivität und Effizienz der Labormedizin. Es ist sicher so, dass das Labor einen grossen Teil der harten Diagnosen liefert. Dies durch mehr und mehr hochspezifische Tests. Aber auch in der Frühdiagnostik bzw. Risikoerfassung (Screening) finden sich mehr und mehr sensitive Tests. 
Abbildung 2

Entwicklung der Medizin von einer klinischen in eine präklinische Erfassung von Krankheiten.

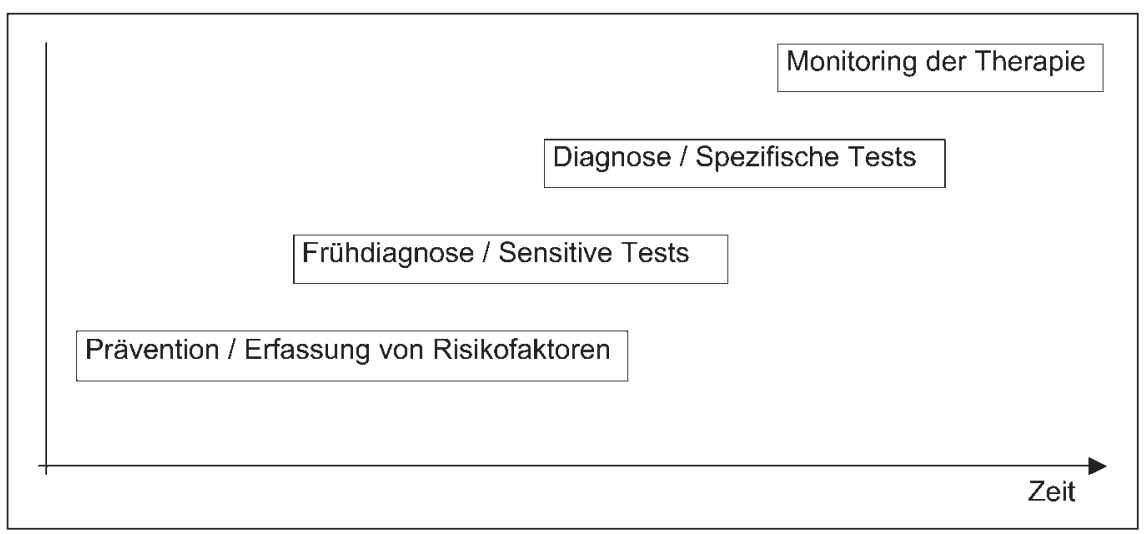

Abbildung 3

Individualisierte Diagnostik und Behandlung.

Überleben = Frühdiagnose und

Abbildung 2 zeigt die Entwicklung der Medizin von einer klinischen in eine präklinische Erfassung von Krankheiten, ja sogar in eine präventive Erfassung von Risiken. Auch im Monitoring von teuren und/oder auch risikoreichen Therapien kommt der Laboranalytik ein zunehmend hoher Stellenwert zu. Bezüglich Effizienz, d.h. der vor allem ökonomischen Aspekte ist zu sagen, dass sowohl für das Praxislabor wie auch für die Grosslaboratorien rationelle und kostengünstige Geräte und Methoden zur Verfügung stehen. Der Preis für eine Glukosebestimmung hat sich in den letzten 20 Jahren dramatisch gesenkt. Auch darf nicht vergessen werden, dass die gesamte Laboranalytik nur etwa 2\% des gesamten Aufwandes im Gesundheitswesen ausmacht! Ein Bruchteil der Ausgaben in der Schweiz für Kosmetika, Strassenverkehr oder andere Luxusgüter. Um die Bedeutung und vor allem die Effizienz der Labordiagnostik zu belegen, hat die SULM zusammen mit renommierten Medizinökonomen in einer Arbeitsgruppe die Wertigkeit von verschiedenen Labortests, wie z.B. D-Dimere-Bestimmung, Messung von BNP und anderen Parametern angegangen, um deren finanziellen Nutzen für die Kostenträger (Krankenkassen) aufzuzeigen.

\section{Ethik}

Auch mit ethischen Fragen befasst sich die Labormedizin und damit auch die SULM. Mit dem Aufkommen von technisch relativ einfach durchführbaren Gentests rücken natürlich ethische Fragen in den Vordergrund. Einen Missbrauch, wie er kürzlich in Form von Inseraten in Zürcher Strassenbahnen zu sehen war, lehnt die SULM entschieden ab. Zusammen mit der Schweizerischen Akademie der Medizinischen Wissenschaft (SAMW), der FAMH und den verschiedenen Fachgesellschaften werden in diesem und im kommenden Jahr mehrere Aktionen lanciert, die solche Missbräuche künftig verhindern sollen. Glücklicherweise sind solche Verstösse in der Schweiz ausgesprochen selten. Die allermeisten Laborleiter, Labormediziner und Laborfachkräfte sind sich der Verantwortung ihrer Tätigkeit und deren Folgen bewusst. Zu einem komplexen oder in der Aussagekraft heiklen Labortest gehört immer eine vorgängige Information und Aufklärung des Patienten durch einen Arzt, gefolgt von einer Information durch den Arzt bei Erhalt des Resultats, inkl. Anleitung über Bedeutung und Grenzen des entsprechenden Tests sowie - falls nötig - Beratung für weiterführende Betreuung. Informationen über die Voraussetzungen für korrekte Probenentnahme und analytisch bedingte Grenzen muss dabei der verantwortliche Laborleiter dem Arzt mitteilen. Selbstdiagnostik oder Diagnostik ohne Beratung macht keinen Sinn. Nicht zu vergessen ist die Bedeutung des Datenschutzes im Zusammenhang mit der Handhabung von Laborresultaten auf allen Stufen.

Zusammenfassend hat sich in den letzten Jahren die Labormedizin von einem simplen Werkzeug oder Hilfsmittel zu einer eigenen Disziplin gemausert (from tool to discipline). 